Dierk Hoffmann und Michael Schwartz (Hrsg.)

Geglückte Integration? 


\section{Schriftenreihe \\ der Vierteljahrshefte für Zeitgeschichte Sondernummer}

Im Auftrag des Instituts für Zeitgeschichte

Herausgegeben von

Karl Dietrich Bracher, Hans-Peter Schwarz, Horst Möller

Redaktion: Jürgen Zarusky

R. Oldenbourg Verlag München 1999 


\title{
Geglückte Integration?
}

\author{
Spezifika und Vergleichbarkeiten \\ der Vertriebenen-Eingliederung in der SBZ/DDR
}

\author{
Herausgegeben von
}

Dierk Hoffmann und Michael Schwartz

R. Oldenbourg Verlag München 1999 
Die Deutsche Bibliothek - CIP-Einheitsaufnahme

[Vierteljahrshefte für Zeitgeschichte / Schriftenreihe]

Schriftenreihe der Vierteljahrshefte für Zeitgeschichte / im Auftr. des Instituts für Zeitgeschichte hrsg. - München : Oldenbourg

Früher Schriftenreihe

Schriftenreihe zu: Vierteljahrshefte für Zeitgeschichte

Sondernr. Geglückte Integration? Spezifika und Vergleichbarkeiten der Vertriebenen-Eingliederung in der SBZ/DDR. - 1999

Geglückte Integration? Spezifika und Vergleichbarkeiten der Vertriebenen-Eingliederung in der SBZ/DDR. - 1999 / hrsg.

von Dierk Hoffmann und Michael Schwartz. - München :

Oldenbourg, 1999

(Schriftenreihe der Vierteljahrshefte für Zeitgeschichte ; Sondernr.)

ISBN 3-486-64503-X

(1) 1999 R. Oldenbourg Verlag GmbH, München

Rosenheimer Straße 145, D-81671 München

Internet: http://www.oldenbourg.de

Das Werk einschließlich aller Abbildungen ist urheberrechtlich geschützt. Jede Verwertung außerhalb der Grenzen des Urheberrechtsgesetzes ist ohne Zustimmung des Verlages unzulässig und strafbar. Dies gilt insbesondere für Verviclfältigungen, Übersetzungen, Mikroverfilmungen und die Einspeicherung und Bearbeitung in elektronischen Systemen.

Gedruckt auf säurefreiem, alterungsbeständigem Papier (chlorfrei gebleicht).

Gesamtherstellung: Appl, Wemding

Bindung: R. Oldenbourg Graphische Betriebe $\mathrm{GmbH}$, München 


\section{Inhaltsverzeichnis}

Dierk Hoffmann/Michael Schwartz

Einleitung

Hermann Graml

Flucht und Vertreibung der Deutschen aus Ostdeutschland und Osteuropa.

Ein Blick auf historische Zusammenhänge

I. Vertriebenen-Integration in Westdeutschland

\section{Sylvia Schraut}

Die westlichen Besatzungsmächte und die deutschen Flüchtlinge

\section{Marita Krauss}

Die Integration Vertriebener am Beispiel Bayerns - Konflikte und Erfolge

Peter Exner

Integration oder Assimilation? Vertriebeneneingliederung

und ländliche Gesellschaft - eine sozialgeschichtliche Mikrostudie

am Beispiel westfälischer Landgemeinden

II. Politisches System und Integrationspolitik in der SBZ/DDR

\section{Manfred Wille}

SED und „Umsiedler“ - Vertriebenenpolitik der Einheitspartei

im ersten Nachkriegsjahrzehnt

\section{Michael Schwartz}

Apparate und Kurswechsel. Zur institutionellen und personellen Dynamik von „Umsiedler“-Politik in der SBZ/DDR 1945-1953

\section{Philipp Ther}

Vertriebenenpolitik in der SBZ/DDR und in Polen 1945 bis 1950

\section{Damian van Melis}

„Angabe nicht möglich“ - Integration statt Entnazifizierung der Flüchtlinge in Mecklenburg-Vorpommern

III. Integrationspolitik und Wirtschaft

\section{Dierk Hoffmann}

Vertriebenenintegration durch Arbeitsmarktlenkung?

Zur Beschäftigungspolitik der SBZ/DDR (1945-1950) 


\section{Arnd Bauerkämper}

Die vorgetäuschte Integration. Die Auswirkungen der Bodenreform und Flüchtlingssiedlung auf die berufliche Eingliederung von Vertriebenen in die Landwirtschaft in Deutschland 1945-1960

Manfred Jahn

Zur sächsischen Spezifik der Aufnahme von vertriebenen Deutschen 1945 bis 1949. Das Fallbeispiel Uranbergbau

IV. Soziale Probleme der Vertriebenen-Integration

\section{Marcel Boldorf}

Fürsorgeunterstützung in Deutschland unter dem Einfluß

der Zwangsmigrationen der Nachkriegszeit (1945-1952)

Michael Grottendieck

Zwischen Integration und Abstoßung. Probleme der Eingliederung von Vertriebenen im münsterländischen Greven sowie von „antifaschistischen Umsiedlern“ im mecklenburgischen Ludwigslust im Vergleich

Steffi Kaltenborn

Wohn- und Lebensverhältnisse von Vertriebenen 1948 in Thüringen

V. Vertreibung und Integration als Lebens-Erfahrung

\section{Ute Schmidt}

"Drei- oder viermal im Leben neu anfangen zu müssen ...“ -

Beobachtungen zur ländlichen Vertriebenenintegration in mecklenburgischen „Bessarabier-Dörfern“

\section{Dagmar Semmelmann}

Zur Integration aus lebensgeschichtlicher Sicht. Eingliederungsverläufe von Flüchtlingen und Vertriebenen in der SBZ/DDR dargestellt am Sonderfall Eisenhüttenstadt

VI. Laufende Forschungsprojekte zur Vertriebenenintegration

Winfried Müller

Zum Forschungsverbund „Die Entwicklung Bayerns durch die Integration der Heimatvertriebenen und Flüchtlinge“

Stefan Donth/Christian Kurzweg/Notker Schrammek/Irina Schwab

Aufnahme und Integration von Flüchtlingen und Vertriebenen in Sachsen von 1945 bis 1952 - Vorstellung eines Forschungsprojekts

Literaturverzeichnis

Abkürzungsverzeichnis

Die Autoren 


\section{Dierk Hoffmann/Michael Scbwartz}

\section{Einleitung}

Alle Besatzungszonen und somit beide 1949 entstandenen deutschen Staaten waren von den Folgen der millionenfachen Flucht, Vertreibung und Zwangsumsiedlung der Deutschen aus Ostdeutschland und Osteuropa vehement betroffen ${ }^{1}$. Beide politischen Systeme reagierten auf diese große soziale Herausforderung mit einer spezifischen Vertriebenen-Integrationspolitik. Anfangs setzten alle Besatzungsregierungen - Sowjets und Westalliierte gleichermaßen - im Bewußtsein der Endgültigkeit jener Massenvertreibung, die sie initiiert oder der sie vor und nach Kriegsende zugestimmt hatten, auf ein weitgehend identisches Integrationskonzept. Dieses sollte einerseits die völlige Gleichberechtigung der Neuankömmlinge mit den Alteingesessenen sichern, andererseits jedoch jedes Sondergruppenbewußtsein der Vertriebenen kanalisieren und abbauen sowie insbesondere Bestrebungen zur politischen Selbstorganisation möglichst unterbinden. Im Zuge des Kalten Krieges veränderte die westliche Vertriebenenpolitik jedoch ihr $\mathrm{Ge}$ sicht: Je mehr die Westalliierten der demokratischen Selbstbestimmung Raum gaben, umso stärker mußte auch den organisierten Interessen der Vertriebenen Rechnung getragen werden. Die Unterdrückung von Selbstorganisation wurde schrittweise bis 1950 aufgehoben. Dies erlaubte den Vertriebenen - um den Preis systemkonformer Mäßigung - eine wirksame Teilnahme nicht nur im Parteien-, sondern auch im Verbändesystem der jungen Bundesrepublik. Diese organisierte Partizipation und die dadurch entfachte politische Konkurrenzsituation trugen erheblich dazu bei, 1952 die lange umstrittene und aufgeschobene Lastenausgleichsgesetzgebung in der Bundesrepublik festzuschreiben. Seither entwickelte sich in Westdeutschland eine langfristige, bis etwa 1970 praktizierte Vertriebenenpolitik, die sich zwischen auf Friedlichkeit festgelegtem Heimkehrpostulat und materieller Integrationserleichterung bewegte. Im Unterschied dazu hielten sowjetische Besatzungsmacht und SED-Hegemonialpartei an der ursprünglichen Assimilationskonzeption aller Alliierten sehr viel deutlicher fest. Überdies betrachteten sie nach einer kurzen Phase gezielter materieller Förderung im Rahmen des DDR-Umsiedlergesetzes von 1950 schon ab etwa 1952/53 die Integration der Vertriebenen - der „ehemaligen Umsiedler" - als weitgehend abgeschlossen. Die politische Durchsetzung dieser voreiligen Sicht wurde durch den Mangel an gesellschaftlichem Pluralismus in der DDR begünstigt. Das Vertriebenen- oder „Umsiedler“-Problem wurde somit in der kontrollierten Öffentlichkeit der DDR nicht nur weitgehend tabui-

${ }^{1}$ Vgl. zu dieser gesellschaftspolitisch brisanten Thematik Theisen, Die Vertreibung der Deutschen; in erstaunlicher, "politisch-korrekter" Scheu vor einer öffentlichen Thematisierung Wolfrum, Zwischen Geschichtsschreibung und Geschichtspolitik; für eine unverkrampfte, Tabuisierungen und Ritualisierungen gleichermaßen benennende wissenschaftliche Aufarbeitung des Verhältnisses von Vertreibungserfahrung und Nachkriegsidentität(en) plädiert Schwartz, Vertreibung und Vergangenheitspolitik. 
siert, sondern auch den allgemeinen gesellschaftlichen Entwicklungsprozessen untergeordnet $^{2}$.

Freilich hatten die gesellschaftlichen Integrationsverläufe selbst in der totalitären DDR nicht nur jenen „überaus repressiven Charakter", der ihnen - zumindest „in weiten Teilen" - immer wieder attestiert wird ${ }^{3}$. Neben die zweifellos vorhandene Repression - die allerdings für Vertriebene auch günstige Wirkungen haben konnte, wie etwa anhand der Profiteure der SBZ-„Bodenreform“ oder der „Entnazifizierung“ gezeigt werden kann ${ }^{4}$ - traten zeitweilig besondere sozialpolitische Integrationsinitiativen, die im erwähnten DDR-Umsiedlergesetz von 1950 kulminierten. Hinzu kamen - ähnlich wie im Westen die generellen Integrationsangebote einer sich entwickelnden Arbeitsgesellschaft mit ihren wachsenden Chancen für sozialen Aufstieg und materiellen Konsum ${ }^{5}$. Zumindest für bestimmte, anpassungswillige Gruppen von Vertriebenen bot der in der DDR diktatorisch forcierte Elitenwechsel in den fünfziger und sechziger Jahren eine im Vergleich zur damaligen westdeutschen Gesellschaft erhöhte soziale Mobilität und deutliche Aufstiegschancen ${ }^{6}$. Aufgrund der alsbald größeren wirtschaftlichen Leistungsfähigkeit und gesellschaftlichen Reformfähigkeit der Bundesrepublik kehrte sich freilich dieses Verhältnis Ende der sechziger/Anfang der siebziger Jahre nachhaltig zugunsten der westlichen Teilgesellschaft um.

Den skizzierten Verläufen der Vertriebenenpolitik in Ost und West entsprachen in gewisser Weise die Konjunkturen der Forschung. Auffallend ist bei der wissenschaftlichen Beschäftigung mit der Vertriebenenproblematik, daß nicht nur in der DDR-Historiographie - wo diese einseitige Ausrichtung wenig überrascht -, sondern auch in der westdeutschen Geschichtsschreibung das Interesse an der Vertriebenen-Integration jenes am vorangegangenen Geschehen der Vertreibung deutlich überwog. Zwar entstand Mitte der fünfziger Jahre eine im Auftrage der Bundesregierung publizierte mehrbändige Dokumentation zur Vertreibung der Deutschen nach $1945^{7}$, doch war nichtsdestoweniger das Erkenntnisinteresse der bundesrepublikanischen Forschung im wesentlichen „der Zukunft zugewandt", wie etwa ein Blick in die resümierende Bibliographie Krallert-Sattlers von 1989 leicht erkennen läßt ${ }^{8}$. Die Geschichte der Vertriebenen-Integration wurde dezidiert als Erfolgsgeschichte der bundesrepublikanischen Gesellschaft geschrieben, um dann seit den sechziger Jahren - parallel zum politischen und gesellschaftlichen Interes-

${ }^{2}$ Vgl. ausführlich in vergleichender Langzeitperspektive: Schwartz, Vertreibung und Vergangenheitspolitik.

${ }^{3}$ Vgl. Wolfrum, Zwischen Geschichtsschreibung und Geschichtspolitik, S. 511; für diesen richtungsweisend Hoffmann / Wille / Meinicke, Flüchtlinge und Vertriebene, S. 15.

${ }^{4} \mathrm{Zu}$ dieser Wirkung der Bodenreform auch Wolfrum, Zwischen Geschichtsschreibung und Geschichtspolitik, S. 512, der freilich die Zahl der dadurch profitierenden „Umsiedler-Bauern“ mit 350000 grob überschätzt; in Wahrheit profitierten etwa 91000 Bauern davon, die mit ihren Familienangehörigen auf die von Wolfrum genannte Zahl gekommen sein dürften; vgl. zur Bodenreform den Beitrag von Arnd Bauerkämper sowie zur Entnazifizierung den Beitrag von Damian van Melis im vorliegenden Band.

${ }^{5}$ Vgl. hierzu Schwartz, Vertreibung und Vergangenheitspolitik, sowie ausführlicher ders., Vom „Flüchtling“ zum Neubürger.

6 Vgl. Dahrendorf, Gesellschaft und Demokratie, S. 453.

7 Vgl. etwa Schieder, Dokumentation der Vertreibung; in neuester Zeit auch die kritisch edierten Erinnerungsberichte von Hübler, Meine Vertreibung aus Prag, sowie von Terpitz, Wege aus dem Osten.

${ }^{8}$ Vgl. Krallert-Sattler, Bibliographie. 
senverlust am Thema - spürbar zu erlahmen. Erst die achtziger Jahre brachten einen erneuten Forschungsschub, der wiederum einige Zeit brauchte, bis er sich von dem „Mythos der raschen Integration“ im Zuge des „Wirtschaftswunders“ (Lüttinger) einigermaßen befreien konnte9. Seither wird der in gewisser Hinsicht durchaus erstaunliche Integrationserfolg zwar nicht hinweggeredet, jedoch auch auf seine Grenzen, Schattenseiten und blinden Stellen hin untersucht. Die Vertriebenen-Integration war gewiß ein Erfolg für das gesellschaftliche und politische System der Bundesrepublik. Dies gilt jedoch nicht in gleicher Weise für alle Vertriebenen. Zumindest eine bemerkenswerte Minderheit älterer Vertriebener der „Erlebnisgeneration“ - insbesondere nicht berufstätige Frauen sowie Angehörige früherer besitzender Schichten - konnten langfristig, nicht selten lebenslang, auf der subjektiven "Verliererseite“ dieses gewaltsamen Umschichtungsprozesses bleiben $^{10}$.

In den achtziger Jahren setzten auch in der SED-kontrollierten Geschichtswissenschaft der DDR Bestrebungen zur Erforschung der „Umsiedler“-Integration ein. Anders als in der westlichen Forschung, die den integrationspolitischen Gründungsmythos der Bundesrepublik in Frage zu stellen lernte, konnte sich die DDR-Geschichtswissenschaft über den SED-Mythos der angeblich spätestens um 1952 erfolgten „Lösung der Umsiedlerfrage" nicht hinwegsetzen ". Gleichwohl signalisierte bereits diese in den engen ideologischen Grenzen des SED-Regimes betriebene Vertriebenenforschung das damals in vielerlei Hinsicht greifbar werdende gesellschaftliche Bedürfnis nach historischer Selbstverzewisserung. In der DDR war diese Trendwende umso bemerkenswerter, als die SEDPolitik schon seit den späten vierziger Jahren eine öffentliche und damit auch eine wissenschaftliche Aufarbeitung der Thematik zu verhindern gewußt hatte. Dies galt nicht zuletzt für eine geplante amtliche Dokumentation der „Umsiedler“-Integrationspolitik, welche die zur SED gehörige Führung der SBZ-„Zentralverwaltung für deutsche Umsiedler “ bereits in der unmittelbaren Nachkriegszeit verwirklicht sehen wollte ${ }^{12}$. An einer terartigen Dokumentation hatte die SED-Führung nach 1948 jedoch ebensowenig Interssse wie an wissenschaftlichen Studien zur „Umsiedler“-Problematik, wie etwa die forschungswillige Berliner Humboldt-Universität im Frühjahr 1950 erfahren mußte ${ }^{13}$.

9 Das Verdienst, diesen Mythos erheblich verunsichert zu haben, gebührt den Studien des Soziologen Paul Lüttinger; vgl. etwa ders., Der Mythos der schnellen Integration, sowie ausführlich ders., Integration der Vertriebenen.

- Vgl. die entsprechende Umfrage bei Sallinger, Die Integration der Heimatvertriebenen.

${ }^{1}$ Vgl. hierzu Wille, Die Lösung der Umsiedlerfrage; Just, Zur Lösung des Umsiedlerproblems; Meinicke, Zur Integration der Umsiedler.

${ }^{2}$ Eine solche Dokumentation wurde in der ZVU bereits im März 1947 erwogen und nach der plötzlichen, politisch bedingten Auflösung der Zentralverwaltung Mitte 1948 von deren früheren Leitern längere Zeit weiter befürwortet; so wandte sich der schon im April 1948 abberufene frühere ZVU-Präsident Rudolf Engel (SED) - zu diesem Zeitpunkt Vizepräsident der Deutschen Verwaltung für Volksbildung - im November 1948 an seinen umsiedlerpolitischen Nachfolger, den früheren ZVU-Vizepräsidenten und damaligen Hauptabteilungsleiter in der Deutschen Verwaltung des Innern, Arthur Vogt (SED), mit der entschiedenen Bitte, den Plan einer solchen Dokumentation weiterzuverfolgen, deren Erstellung seinerzeit noch mit dem zuständigen Vertreter des SED-Zentralsekretariats, Paul Merker, vereinbart worden sei; vgl. hierzu BAB, DO 1/10/1, Bl. 80 f., und DO 1/10/4, Bl. 62; Vogt, der seinerseits im Juni 1949 seine Funktion verlor, setzte sich noch in den späten fünfziger Jahren SED-intern erfolglos für eine Wiederaufnahme dieser Pläne ein; vgl. hierzu den Beitrag von Michael Schwartz im vorliegenden Band.

3 Vgl. MdI DDR, Abteilung Bevölkerungspolitik an Staatssekretär Warnke, Notiz über eine Besprechung mit SED-Zentralsekretariatsabteilungsleiter Plenikowski, ca. Mai 1950; in: BAB, DO 1/5512. 
Die mit der politischen Entwicklung seit 1989/90 gegebene neue Forschungslandschaft hat die empirischen Arbeitsmöglichkeiten im Bereich der DDR-Geschichte entscheidend verbessert. Auch die Vertriebenen-Integrationspolitik ist - wie nicht zuletzt die Beiträge des vorliegenden Bandes dokumentieren - ein solches prosperierendes Forschungsfeld. Das auf der Entwicklung von Vertriebenenpolitik und gesellschaftlicher Vertriebenenintegration in der SBZ/DDR liegende Schwergewicht des Bandes resultiert nicht unwesentlich aus diesem forschungsgeschichtlich gegebenen Nachholbedarf. Zugleich werden durch einen wachsenden Kreis interessierter Wissenschaftler aus unterschiedlichen Disziplinen den neuen Möglichkeiten neue Fragestellungen hinzugefügt.

Immer stärker tritt dabei die Notwendigkeit hervor, über die nunmehr parallele zeithistorische Forschung zur geteilten deutschen Nachkriegsgeschichte allmählich zu einer gesamtdeutschen Perspektive zu gelangen. Die zeithistorische Forschung "hat es im Fall Deutschland nach 1945 mit zwei verschiedenen ,Zeitgeschichten' zu tun“, ja sogar mit mindestens dreien, wenn die gemeinsame „Vorgeschichte“ der Weimarer Republik und der NS-Diktatur Berücksichtigung finden sollen. Das zentrale Forschungsvorhaben der Zukunft wird folglich darin bestehen, „diese Grenzen aufzuheben und die verschiedenen deutschen ,Zeitgeschichten' [...] in ein ,Relationengefüge“ (H. G. Hockerts) zu bringen bzw. - in durchaus Hegelschem Sinn - in der einen deutschen Zeitgeschichte ,aufzuheben “"14. Das bedeutet, sowohl die Beziehungsgeschichte des selbst in Teilung und Konfrontation vielfältig aufeinander bezogenen Gesamtdeutschland als auch die auf Ähnlichkeiten und Unterschiede gleichermaßen abhebende vergleichende Metbode ernstzunehmen. Nicht zuletzt im Forschungsfeld der Vertriebenenintegration bestätigt sich die Beobachtung, daß „komparative Geschichte und Beziehungsgeschichte [...] nicht dasselbe, aber durchaus kompatibel und verknüpfbar sind “15.

Pragmatische Skepsis gegenüber einer allzu ausgreifenden und vorschnellen Euphorie hinsichtlich umfassender Vergleiche ist jedoch durchaus angebracht ${ }^{16}$. So überwiegt auch im vorliegenden Band - neben verschiedentlicher Erprobung der vergleichenden Perspektive in einigen Beiträgen - ganz bewußt die Konzentration auf die Entwicklung in der SBZ/DDR. In vielen Bereichen auch unserer Thematik gilt es nach wie vor, den zweiten Schritt nicht vor dem ersten zu tun. Selbst ein begrenzter Vergleich bedarf, wenn er mehr sein will als die Selbstbestätigung seiner erkenntnisleitenden Prämissen, deren fortgesetzter Verunsicherung durch die Empirie. Das Ziel einer Verknüpfung der Zeitgeschichten der DDR und der Bundesrepublik geht daher unerläßlich mit der Einsicht in die Notwendigkeit einher, zunächst „die innere Geschichte der DDR und die Eigenart ihres politischen Systems intensiv anhand der nunmehr verfügbaren Archivalien zu untersuchen“, damit „erst einmal das Ungleichgewicht der Kenntnisse über den westlichen und östlichen Teil Deutschlands verringert wird “17. Erst auf dieser Basis können systembedingte Spezifika wie auch gesamtdeutsche Vergleichbarkeiten der SBZ/DDR-Entwicklung klar konturiert und gewichtet werden. Unter diesen Voraussetzungen vereint der vorliegende Sammelband verschiedene Forschungsfelder und -ergebnisse, die stärker miteinander vernetzt werden sollten. Es sind dies unter anderem

${ }_{14}$ Vgl. Möller / Mehringer, Die Außenstelle Potsdam des Institutes für Zeitgeschichte, S. 174.

15 Vgl. Kocka, Die Geschichte der DDR, S. 16.

16 Vgl. ebenda.

17 Vgl. Doering-Manteuffel, Deutsche Zeitgeschichte, S. 28. 
- die Rekonstruktion von Vertriebenenpolitik auf unterschiedlichen staatlichen Handlungsebenen; wichtig erscheint zum einen eine umfassende Geschichte des mit Vertriebenenpolitik befaßten politischen Teil-Systems, die sich nicht auf die Geschichte der Sonderverwaltungen beschränken kann, sondern möglichst sämtliche relevanten Institutionen und Personengruppen einzubeziehen hat. Zum anderen sollte der Versuch unternommen werden, die gesellschaftlichen Rückkoppelungen einer solcherart konzipierten und umgesetzten Integrationspolitik in den Blick zu nehmen;

- eine verschränkte Politik- und Sozialgeschichte der Integration auf der Mikroebene, die über Kreis- und Lokalstudien die Resultate und Begrenzungen zentraler Politikvorgaben, aber auch die Eigendynamik gesellschaftlicher bzw. lebensweltlicher Prozesse zu beschreiben sucht;

- soziologische Langzeituntersuchungen, die mit quantitativen Methoden bisher allein für die Bundesrepublik möglich waren, wo aus politischen Gründen eine besondere Vertriebenenstatistik über Jahrzehnte weitergeführt wurde, während die DDR - ebenfalls aus politischen Gründen - solche statistischen Bilanzierungen bereits 1949 abbrach; dennoch verspricht auch hier eine Verknüpfung von soziologischen und erfahrungsgeschichtlichen Methoden weiterführende Ergebnisse;

- erfahrungsgeschichtliche Studien durch lebensgeschichtliche Interviews, die sowohl die Rolle der Vertreibungserfahrung als biographische Zäsur beleuchten als auch die Schritte der allmählichen Integration in die neuen Lebensverhältnisse der Nachkriegszeit in den Blick nehmen.

Der erste Teil des vorliegenden Bandes beschäftigt sich mit der - im Vergleich zur SBZ/DDR weitaus besser erforschten - Vertriebenen-Integration in Westdeutschland, also der Entwicklung in den westlichen Besatzungszonen und der späteren Bundesrepublik. Auf unterschiedlichen Ebenen - die zentralen Besatzungsbehörden, eine Region und drei Lokalgesellschaften betreffend - werden zentrale Ergebnisse der bisherigen "West“-Forschung resümiert. Sylvia Schraut, Mannheim, behandelt das Verhältnis der amerikanischen und britischen Besatzungsmächte zu den in ihre Zonen gelangenden deutschen Flüchtlingen und Vertriebenen. Gemeinsamkeiten und Unterschiede der Vertriebenenpolitik beider angelsächsischen Besatzungsmächte werden herausgearbeitet, wobei insbesondere für die amerikanische Militärverwaltung eine differenzierte Sichtweise präsentiert wird: Die Flüchtlingsfrage, die gerade in den Besatzungsjahren engstens mit der Politik der vier Siegermächte verknüpft war, stellte sich zunächst überwiegend als Transportproblem dar und bedurfte insgesamt einer großen Koordinationsanstrengung zwischen alliierten und deutschen Verwaltungen. Schraut skizziert anschließend die amerikanische sowie die britische Politik gegenüber den Flüchtlingen und Vertriebenen. Unterschiedliche Vorgehensweisen gab es etwa in der Frage des Aufbaus von besonderen Flüchtlingsverwaltungen, in der Frage politischer Beteiligung der Deutschen sowie bei der Hierarchisierung assimilationsfördernder Maßnahmen. Ein letztlich erfolgloser Versuch zur Beilegung dieser interalliierten Differenzen stellte der Ende 1948 eingerichtete trizonale Arbeitskreis "Tripartite working party on german refugees" dar, dessen GrünJung auf die Initiative der britischen Militärregierung zurückging. Eine einheitliche Veririebenenpolitik im Westen Deutschlands konnte deswegen erst nach Gründung der Bundesrepublik beginnen.

Die Flüchtlingsintegration in Bayern steht in mancher Hinsicht exemplarisch für die Entwicklung in den Ländern der westlichen Besatzungszonen. Dies ist nicht überraschend, ging es doch zunächst - mit Varianten - überall um die gleichen Schritte von not- 
dürftiger Aufnahme zu vertiefter wirtschaftlicher, sozialer und kultureller Eingliederung. Dennoch gibt es manche bayerische Spezifika, die Marita Krauss, Bremen, untersucht hat. Zu diesen zählt etwa die vergleichsweise größere Toleranz der amerikanischen Besatzungsmacht gegenüber der geschlossenen Ansiedlung heimatvertriebener Spezialindustrien, durch deren kräftig mit Krediten unterstützten Aufbau Bayern einen nicht unerheblichen Industrialisierungsschub gerade in bislang strukturschwachen ländlichen $\mathrm{Ge}$ bieten erlebte. Auch in anderen Bereichen trugen die Flüchtlinge dazu bei, das Land zu modernisieren und zu differenzieren: Dies gilt für die politische Kultur ebenso wie für Volksbildung oder konfessionelle Durchmischung. Konzepten der „künstlichen“ Staatsintegration des 19. Jahrhunderts entlehnt war Krauss zufolge die Ernennung der Sudetendeutschen, der größten Vertriebenengruppe in Bayern, zum „vierten Stamm“ Bayerns, womit die Aufnahmegesellschaft die kulturelle Identität der Vertriebenen zugleich annahm und vereinnahmte. Die spezifische Mischung von Traditionalismus und Modernität, die hier manifest wird, war charakteristisch für das Bayern der Nachkriegszeit.

Unter der Fragestellung "Integration oder Assimilation“ behandelt Peter Exner, Marburg, die Vertriebenen-Eingliederung in der ländlichen Gesellschaft am Beispiel einiger westfälischer Landgemeinden. Dabei wird deutlich, daß die erforderliche „Eingliederung" der Vertriebenen, die zeitweise nahezu ein Drittel der Bewohner der untersuchten Landgemeinden ausmachten, diese Dörfer (Ottmarsbocholt, Heek und Rödinghausen) vor tiefgreifende materielle und mentale Belastungsproben stellte. Althergebrachte Strukturen gerieten mit der Flüchtlingsansiedlung ins Wanken. Entsprechend defensiv reagierte das „einheimische“ Milieu: Besonders wenn Flüchtlinge in großer Zahl dem Sozialraum Dorf zugewiesen wurden, was bei der Mehrheit der Einheimischen Bedrohungsgefühle hervorrief, war eine anfänglich starre Frontbildung zwischen den beiden Gruppen unvermeidlich. Die materielle Notlage der „Trümmergesellschaft" (Teppe) verschärfte gegenseitige Irritation und Ablehnung weiter, die vorrangig auf unterschiedlichen Orientierungs- und Verhaltensweisen basierten. Die Neuankömmlinge galten in ihrer neuen Umgebung als „Habenichtse“, da sie weder soziales noch materielles Kapital vorweisen konnten, das für ihre Anerkennung in den von besitzhierarchischen Wertmustern geprägten Dorfgesellschaften erforderlich gewesen wäre. Die gegenseitige Abgrenzung sorgte zum einen für eine Koexistenz zweier sich größtenteils unvermittelt und unverbunden gegenüberstehender Sozialgruppen, behinderte zum anderen jedoch eine reibungslose und rasche Eingliederung der Neubürger, die sich nicht als Integration, d. h. als wechselseitiger Prozeß der Angleichung beider sozialen Gruppen, sondern als allmähliche und vor allem einseitige Anpassung der Vertriebenen vollzog. Anfängliche Abgrenzung und allmähliche Assimilation vollzogen sich gleichermaßen in zentralen Bereichen dörflichen Lebens: In den Verteilungskämpfen um die raren Ressourcen der Landgemeinden, der wirtschaftlichen Eingliederung der Flüchtlingsbauern, der Mitwirkung an den politischen Entscheidungsprozessen, der Partnerwahl sowie dem Fest- und Vereinsleben.

Der zweite Teil des Bandes eröffnet das Schwerpunktthema der Vertriebenenintegration in der SBZ/DDR und konzentriert sich dabei auf das dortige politische System und dessen Integrationspolitik. Manfred Wille, Magdeburg, diskutiert in seinem Beitrag die inhaltlichen Grundlinien der Vertriebenenpolitik der SED im ersten Nachkriegsjahrzehnt. Dabei macht er auf das spannungsreiche Verhältnis zwischen KPD und sowjetischer Besatzungsmacht unmittelbar nach Kriegsende aufmerksam. Die Vertreibung von Deutschen aus den ehemals deutschen Ostgebieten war beispielsweise für Wilhelm Pieck 
ınfangs keineswegs selbstverständlich und wurde deutlich in Frage gestellt. Ansonsten 1ahm sich die KPD-Führung der Flüchtlingsfrage allerdings nur reaktiv-unsystematisch and halbherzig an; Vertriebenenpolitik besaß innerhalb des Aufgabenkataloges der KPD-Führung einen eher untergeordneten Rang. Erst im Vorfeld der Wahlen von 1946 anden die Vertriebenen verstärkte Aufmerksamkeit bei der nunmehrigen SED. Wille nacht deutlich, daß die Haltung des SED-Parteivorstandes auch zur Oder-Neiße-Grenze zunächst durchaus zwiespältig war: Während eine Gruppe um die beiden Vorsitzenlen Wilhelm Pieck und Otto Grotewohl noch an mögliche Korrekturen und Revisionen zeglaubt habe, hätten sich andere - vor allem Walter Ulbricht - rasch mit dem von Stalin zeschaffenen status quo abgefunden. Erst ab 1947 habe die SED aufgrund außen- und inrenpolitischer Faktoren ihre Haltung in der „Umsiedlerfrage“ nachhaltig geändert: Die Aussöhnung mit den „sozialistischen Bruderstaaten“ wurde vorrangig und die bis dahin 1och mögliche Diskussion über die Grenzfrage daher entsprechend radikal unterbunden. Jamit begann eine Phase, an deren Ende nach dem Willen der SED-Führung schon Anang der fünfziger Jahre der erfolgreiche Abschluß der Vertriebeneneingliederung stehen iollte. Wille zeigt, daß diese doktrinäre Position, die jeder Realität Hohn sprach, auch zu Auseinandersetzungen der SED mit den bürgerlichen Parteien (CDU und LDPD) ührte.

Michael Schwartz, IfZ-Außenstelle Berlin, beleuchtet das Problem der Vertriebeneninegration unter politikgeschichtlichem Blickwinkel. Unter der zentralen Fragestellung, welche grundlegenden Ziele diese Integrationspolitik verfolgte, auf welche Weise sie sonkret operationalisiert wurde und welche politisch-administrativen Institutionen mit ler Umsetzung solcher Politik befaßt waren, steht das Verhältnis von „Apparaten und Kurswechsel“ in der "Umsiedler"-Politik der SBZ/DDR zwischen 1945 und 1953 im Mittelpunkt. Die „institutionelle und personelle Dynamik“ in der Entwicklung der beeiligten „Apparate“ gewann auch in inhaltlicher Hinsicht besondere Bedeutung für den Verlauf der Flüchtlings-Integrationspolitik der SBZ/DDR. Deutlich wird dabei, daß Kontinuitäten und Diskontinuitäten der „Umsiedler“-Politik nicht allein auf politische Anweisungen aus Moskau oder auf Entscheidungen der SED-Spitze um Walter Ulbricht, io maßgeblich diese letztlich waren, zurückzuführen sind. Gerade während des Besterens der SBZ, aber auch noch in den ersten Jahren der DDR wurde die inhaltliche und nstitutionelle Ausgestaltung dieser Politik wesentlich auch durch ein personelles Netzverk von Funktionären in den Fachabteilungen der Sowjetischen Militäradministration, les zentralen Parteiapparates der SED sowie - und hier fast ausschließlich von SED-Verretern - relevanter staatlicher Verwaltungen beeinflußt und mitgestaltet, die damit gevissermaßen als „Geschäftsführer“ der „Umsiedler“-Integrationspolitik fungierten. Dieies personelle Netzwerk, das sich zwischen 1945 und 1947 herausgebildet und zunehnend professionalisiert hatte, überdauerte die in der Forschung häufig allzu stark betone inhaltliche und institutionelle Zäsur des Jahres 1948 und trug gerade dadurch dazu bei, laß die „Umsiedler“-Politik selbst noch einige Jahre vordergründig ihren Stellenwert bevielt. Das im September 1950 öffentlichkeitswirksam verkündete „Umsiedlergesetz“ der JDR bildete jedoch nicht nur den Höhe-, sondern zugleich auch den Schlußpunkt dieser solitik, die etwa 1953 auslief.

In der Forschung zur Geschichte der SBZ/DDR sind Vergleiche mit anderen osteurosäischen Staaten bisher ein Desiderat geblieben. Ein Vergleich der SBZ/DDR mit der Jolksrepublik Polen, wie ihn Philipp Ther, Berlin, anstellt, scheint auch deshalb nahelie;end, weil beide Staaten im Kontext sowjetischer Hegemonie in ihren Anfangsjahren 
mit einer massenhaften Zwangsmigration und daraus resultierenden ähnlichen Integrationsproblemen konfrontiert waren. Die SBZ/DDR und Polen wollten beide eine möglichst rasche Integration. Den zentralen Unterschied der jeweiligen Vertriebenenpolitik erblickt Ther darin, daß diese in Ostdeutschland bis 1950 egalitär geprägt gewesen sei, während sie in Polen unter einem nationalistischen Leitmotiv gestanden habe. Drei inhaltliche Ansätze integrativer Vertriebenenpolitik werden vorgestellt: ein sozialkaritativer, ein redistributiver und ein sozialrevolutionärer. Die Durchführung dieser Politik stieß in beiden Ländern auf zahlreiche Schwierigkeiten. Ther macht dafür die begrenzte Wirksamkeit der jeweiligen Vertriebenenverwaltung, die Unterrepräsentierung der Vertriebenen und ihrer Interessen im politisch-administrativen System sowie die Monopolisierung des gesellschaftlichen Lebens durch Staat und (kommunistische) Partei verantwortlich. Im Ergebnis sei daher die staatliche Vertriebenenpolitik in beiden Ländern gescheitert, was indirekt die Grenzen beider Regimes markiere. Die Vertriebenen seien bis 1950 eine deutlich unterprivilegierte Bevölkerungsgruppe geblieben, und ihre dennoch fortschreitende Integration sei daher mehr beiläufige Folge der industriellen Entwicklung und der daraus folgenden Urbanisierung.

Auf besondere strukturelle Problemlagen und daraus folgende politische Konsequenzen in Mecklenburg-Vorpommern macht Damian van Melis, Düsseldorf, aufmerksam. Ihn interessiert der Zielkonflikt zweier zentraler politischer Aufgaben: Vertriebenenintegration und Entnazifizierung. Die Vertriebenen machten im nördlichsten Land der SBZ fast die Hälfte der Bevölkerung aus; kein Bereich des politischen und gesellschaftlichen Lebens blieb von ihren Problemen unberührt. Während die Flüchtlingspolitik aber gesellschaftliche Integration intendierte, zielte die Entnazifizierung im Gegenteil auf politische Desintegration der ehemaligen NSDAP-Mitglieder, deren Anteil auch unter den Vertriebenen entsprechend hoch war. Obwohl eine Reihe von Politikern die unzureichende Entnazifizierung der Flüchtlinge monierten, war das kommunistisch dominierte Landespersonalamt bestrebt, die Thematik „Entnazifizierung der Umsiedler" von der politischen Tagesordnung möglichst fernzuhalten. Somit konnten zahlreiche NS-belastete Ortsfremde ungeprüft und unerkannt in den neu geordneten öffentlichen Dienst übernommen werden. Während die Vertriebenen im allgemeinen zu den am stärksten benachteiligten sozialen Gruppen gehörten, blieben sie von der Entnazifizierung paradoxerweise weitgehend verschont. Eine entscheidende Voraussetzung dafür war allerdings die ausdrückliche Loyalität gegenüber der Besatzungsmacht und der KPD/SED. Die Entnazifizierung - so van Melis - diente dem Elitenwechsel und dem Umbau der Verwaltungsund Herrschaftsstrukturen, und im Mittelpunkt des Interesses der Entscheidungsträger stand dabei weniger die personelle Bereinigung der Vergangenheit als vielmehr die gesellschaftliche Neuordnung.

Der dritte Teil des Bandes konzentriert sich auf den Zusammenhang von Integrationspolitik und Wirtschaftsentwicklung in der SBZ/DDR. Auf die große Bedeutung der Arbeitsplatzbeschaffung für die Integration der Flüchtlinge und Vertriebenen verweist Dierk Hoffmann, IfZ-Außenstelle Berlin. Er behandelt vor allem die Probleme der staatlichen Steuerung des "Arbeitsmarktes" in der SBZ/DDR nach 1945 und unterstreicht die Bedeutung der einzelnen Vertreibungsphasen für die Integration in den Arbeitsmarkt. Erst spät wurden Aufnahme- und Quarantänelager errichtet, in denen die Neuankömmlinge hinsichtlich Arbeitsfähigkeit und Einsatzmöglichkeit untersucht und - im Idealfall - umgehend in die Aufnahmegebiete weitergeleitet werden sollten. Das ursprüngliche Vorhaben, die Eingliederung in den Arbeitsmarkt von der Nachfragesituation vor Ort 
sowie von der beruflichen Qualifikation abhängig zu machen, erwies sich als Makulatur, da die amtliche Registrierung der „Umsiedler“ erst einsetzte, als sich Millionen von Vertriebenen längst - gewissermaßen „planlos“ - über das Gebiet der SBZ verteilt hatten. Auch aufgrund der Binnen- und Westwanderungen war eine planvolle Ansiedlung nahezu unmöglich. Hoffmann macht zudem deutlich, daß die beruflichen Chancen der „Umsiedler" höchst unterschiedlich waren und stark von den lokalen Gegebenheiten auf dem Arbeitsmarkt abhingen. Insgesamt ist eine Benachteiligung gegenüber der eingesessenen Bevölkerung nicht zu übersehen. Darüber hinaus muß die Eingliederung der Vertriebenen in den ostdeutschen Arbeitsmarkt im Kontext der staatlichen Steuerung der Gesamtwirtschaft gesehen werden, die erst allmählich aufgebaut werden konnte. Dies war ein Grund dafür, warum die Zentral- und Landesverwaltungen, von einigen Ausnahmen abgesehen, keine gesonderten Förderprogramme für „Umsiedler“ ausgearbeitet hatten. Flüchtlinge und Vertriebene waren für die Arbeitsverwaltungen nur eine von vielen Personengruppen, die in den Arbeitsmarkt integriert werden mußten. Außerdem wollte die SED-Führung jegliches Sonderbewußtsein der „Umsiedler“ unterdrücken. Ferner befanden sich Arbeits- und „Umsiedler“-Verwaltungen in einem jahrelangen Konkurrenzverhältnis, das möglicherweise zu zeitlichen Verzögerungen etwa bei der Ausarbeitung arbeitsrechtlicher Bestimmungen führte und mit ein Grund für die Auflösung der ,Umsiedler'-Sonderverwaltung im Jahre 1948 gewesen sein dürfte.

Mit der bewußt provokativen Formel der „vorgetäuschten Integration“ stellt Arnd Bauerkämper, Potsdam, die geläufige positive Würdigung der SBZ-Bodenreform für die Vertriebenenintegration in Frage. Dies geschieht im Rahmen eines gesamtdeutschen Vergleichs-Ansatzes, der sich auf die Auswirkungen der SBZ-Bodenreform konzentriert, in diesem Zusammenhang jedoch auch die Bedeutung der westdeutschen Flüchtlingssiedlungspolitik für die berufliche Eingliederung von Vertriebenen in die Landwirtschaft in den Jahren 1945-1960 thematisiert. Dabei macht Bauerkämper zunächst die Parallelität der Problemlagen deutlich: $\mathrm{Da}$ sich die Flüchtlinge aus den verlorenen Ostprovinzen in der unmittelbaren Nachkriegszeit vor allem in ländlichen Gebieten niederließen, erschien eine berufliche Integration der Vertriebenen in die dortige Agrarwirtschaft unumgänglich. Die umfangreiche „Bodenreform“, die in der Sowjetischen Besatzungszone schon 1945 herbeigeführt wurde, um die Herrschaft der KPD auf dem Lande durchzusetzen, eröffnete auch vielen „Umsiedlern“ zunächst die Chance einer Existenzgründung. Zahlreiche in diesem Rahmen entstandene Neubauernbetriebe konnten jedoch trotz erheblicher materieller und finanzieller Unterstützung von staatlicher Seite ökonomisch nicht dauerhaft gesichert werden. In Westdeutschland hingegen scheiterte eine von den Besatzungsmächten zunächst durchaus favorisierte Bodenreform im eskalierenden Ost-WestKonflikt; die Länderregierungen und das Bundeskabinett förderten aber mit erheblichen Mitteln eine eher an Weimarer Vorbildern orientierte Politik bäuerlicher Siedlung. Nur wenigen Vertriebenen gelang jedoch die Gründung rentabler Bauernbetriebe. Mit dem Wiederaufbau der Industrie in den fünfziger Jahren wurde die Agrar-Ansiedlung der Flüchtlinge in beiden deutschen Staaten schließlich weitgehend obsolet. In der DDR wurden die Neubauernbetriebe in die Landwirtschaftlichen Produktionsgenossenschaften integriert, während der Strukturwandel in der westdeutschen Landwirtschaft auch die vergleichsweise nur in geringer Zahl angesiedelten Vertriebenen erfaßte. Bauerkämper bietet hierbei eine vergleichende Makroperspektive für die mikrohistorischen Ergebnisse Exners und zeigt, daß in beiden deutschen Staaten auch die gesellschaftliche Eingliederung der Flüchtlinge auf dem Lande bis in die späten fünfziger Jahren nur unvoll- 
ständig gelang. Zudem verweist der Beitrag auf das Verhältnis von Flüchtlingsintegration und sozioökonomischer Transformation als Faktor der wechselseitigen Abgrenzung in der Systemkonkurrenz zwischen der Bundesrepublik und der DDR bis 1960.

Manfred Jahn, Dresden, behandelt am sektoralen Beispiel des Uran-Bergbaus in der SBZ regionale Besonderheiten der Vertriebenenintegration in Sachsen. Er geht dabei von der Annahme aus, daß der ab etwa 1947 expandierende Uranerzbergbau die Aufnahme von vertriebenen Deutschen im Land Sachsen in ganz spezifischer Weise geprägt habe. Die Vertriebenen, die den Ansiedlungsdirektiven sowohl der SMAD bzw. SMAS und der mit Sondervollmachten ausgestatteten sowjetischen Wismut AG als auch der sächsischen Umsiedlerbehörde unterworfen waren, bildeten eine attraktive Zielgruppe für den Uran-Bergbau hinsichtlich Arbeitskräftewerbung und -lenkung, wobei die Wismut AG sich bei der forcierten Arbeitskräftelenkung in den Bergbau durchsetzen konnte. Die massive Ansiedlung von Vertriebenen in dem von akutem Wohnraummangel gekennzeichneten Wismut-Gebiet zielte dabei auch auf die Eindämmung der hohen Fluktuation der hier bereits arbeitsverpflichteten Beschäftigten $a b$ und hatte zum Teil eine Zwangsumsiedlung nicht im Uranbergbau tätiger alteingesessener Bevölkerung sowie nicht einsatzfähiger Vertriebener in andere Gebiete zur Folge. Anhand eines Vergleichs mit der Zuwanderung von Zwangsmigranten in das Braunkohlenrevier Borna (Sachsen) verdeutlicht Jahn Gemeinsamkeiten und Unterschiede der beiden Bergbau-Typen. Dabei gelangt er zu dem Schluß, daß der forcierte Wirtschaftsaufbau in den Bergbaugebieten, das materielle Privilegiensystem im Uran- und Kohlebergbau sowie die berufsbezogene Integration von Vertriebenen offensichtlich zu einer schnelleren dauerhaften Seßhaftwerdung führte, als dies in wirtschaftlich anders geprägten Regionen der Fall war. Angesichts der unbefriedigenden Quellenlage in sächsischen Archiven bedürfen Fragen nach gesellschaftlichen Konflikten bei der Eingliederung von Vertriebenen, nach Wandlungen der Bevölkerungsstrukturen und lokalen Integrationsbedingungen allerdings noch weiterer Anstrengungen.

Der vierte Teil des Bandes behandelt soziale Probleme der Vertriebenen-Integration. Marcel Boldorf, Mannheim, untersucht in Form eines gesamtdeutschen Vergleichs den Einfluß der durch Flucht und Vertreibung ausgelösten millionenfachen Zwangsmigrationen auf die Entwicklung der Fürsorgeunterstützung in beiden entstehenden deutschen Staaten zwischen 1945 und 1952. Dabei wird deutlich, daß soziale Entwurzelung und Depossedierung vieler Vertriebener die Hauptursache für den überdurchschnittlich hohen Unterstütztenanteil innerhalb der Flüchtlingsbevölkerung in allen vier Besatzungszonen gewesen ist. Die primäre Ansiedlung von Vertriebenen in ländlichen Gebieten, die zwar der Ernährungs- und Wohnraumversorgung, nicht aber den Erfordernissen des Arbeitsmarktes entsprach, wirkte sich in allen Zonen negativ aus. Umso schwerer wiegt hinsichtlich der alltäglichen Lebenssituation vieler bedürftiger Vertriebener die Feststellung Boldorfs, daß manche wichtige Zuweisungsgebiete wegen dort ungenügender Durchsetzung des Bedarfsprinzips gerade nicht die höchsten Unterstütztenquoten aufwiesen. Auch für die Schlußphase seines Untersuchungszeitraumes erkennt Boldorf eher gesamtdeutsche Parallelen: Sieht man von den Sonderfürsorgebereichen ab, die in der Bundesrepublik für gewisse Gruppen von Vertriebenen geschaffen wurden, herrschte für die Unterstützungsempfänger in beiden Teilen Deutschlands um 1950 ein annähernd gleich dürftiger materieller Lebensstandard.

$\mathrm{Daß}$ sich soziale Probleme keineswegs in der Sozialfürsorge erschöpften, sondern in vielfältigen lebensweltlichen Konflikten zwischen Vertriebenen und Einheimischen vor 
Ort ihren Ausdruck fanden, verdeutlicht in wiederum vergleichender Perspektive Michael Grottendieck, Münster, anhand der lokalen Integrationsbedingungen und -konflikte für Vertriebene in einer mecklenburgischen (Ludwigslust) und einer münsterländischen Gemeinde (Greven). In beiden deutschen Nachkriegsgesellschaften entstand ab 1945 aufgrund des Vertriebenenzustroms ein immenses Konfliktpotential, das sich im wesentlichen in soziokulturellen Konflikten sowie in Integrations- und Abstoßungsprozessen zwischen Alteingesessenen und Hinzugekommenen entlud. Detailliert werden in Ludwigslust sowie in Greven Entstehung, Form und Verlauf der Konflikte um die Anrechte der Vertriebenen auf Wohnung, Arbeitsplätze, Gebrauchsgegenstände und Hilfsmaßnahmen beschrieben. In Greven gewann dieser Konflikt seine besondere Dimension dadurch, daß sich auch alteingesessene Grevener Familien in einer ähnlich mißlichen Lage wie die Vertriebenen befanden, da sie aufgrund der Einrichtung eines zentralen Sammellagers für ausländische Fremdarbeiter für zwei Jahre ihre Häuser hatten räumen müssen. Doch konnten diese teil-enteigneten Alteingesessenen im Unterschied zu den Vertriebenen mit ausgesprochen großer Hilfsbereitschaft der übrigen Altbewohner des Dorfes rechnen. Diese Hilfeleistungen gaben Anlaß für die besonderen Konflikte der Vertriebenenintegration in Greven. In Ludwigslust wiederum stellten Probleme bei der Aufnahme sogenannter „antifaschistischer Umsiedler“ aus dem Sudetenland einen Sonderfaktor dar, deren hoher SED-konformer politisch-moralischer Status ihrer Eingliederung eine besondere Dringlichkeit verlieh. Grottendieck zeigt eindringlich, daß die Konflikte in Ludwigslust nicht nur aus politisch-kulturellen Unterschieden oder überzogenen Ansprüchen dieser privilegierten „Umsiedler“-Gruppe resultierten, sondern auch dadurch bestimmt wurden, daß die neuen kommunistischen Machthaber in der SBZ die Eingliederung selbst dieser „politisch korrekten“ Gruppe von Neuankömmlingen nicht mit dem eigentlich zu erwartenden Nachdruck betrieben - und wohl auch nicht betreiben konnten, wollten sie nicht ihre Macht in der (alteingesessenen) sozialen Umgebung aufs Spiel setzen. Mochten bei der Ankunft „antifaschistischer Umsiedler“ das vor Ort Machbare sowie das aus politischen Erwägungen Wünschbare stärker aufeinanderprallen als bei der Aufnahme vermeintlich „normaler“ Vertriebener, so wirft dieser Umstand doch ein bezeichnendes Licht auf die allgemeine Integrationssituation in der SBZ/DDR. Grottendieck sieht gerade in diesen vor Ort nicht überwindbaren Problemen einen wesentlichen Grund für jenen abrupten Kurswechsel, der im Jahre 1948 in der „Umsiedler“-Politik der SBZ erfolgte.

Ein zentrales Problemfeld des Vertriebenen-Alltags in allen Zonen untersucht Steffi Kaltenborn, Magdeburg, mit ihrer auf das Land Thüringen konzentrierten Momentaufnahme der "Wohn- und Lebensverhältnisse“ der dort untergebrachten Vertriebenen im Jahre 1948. Mit der Frage der Aufteilung der knappen Wohnraum-Ressourcen berührt sie einen Konfliktherd zwischen Vertriebenen und Alteingesessenen, der lange Jahre die alltägliche Lebenssituation der Vertriebenen maßgeblich mitbestimmte. Dabei wird am Beispiel Thüringens - hier waren, gegenüber anderen Regionen, sowohl die Kriegsschäden wie andererseits der Anteil der Vertriebenen an der Nachkriegsbevölkerung relativ gering - deutlich, daß die Wohnraum-Verteilungsfrage in der unmittelbaren Nachkriegszeit auf administrativem Weg kaum lösbar war; zum einen waren die Ressourcen objektiv knapp, zum anderen wurden die Vertriebenen durch die Machtkonstellationen vor Ort in der Regel deutlich benachteiligt: Den Wohnraumbesitzern gelang es - ungeachtet geltender Gesetze und behördlicher Anordnungen und nicht selten unter Duldung durch lokale Amtsträger - immer wieder erfolgreich, sich gegen 
die Beschlagnahme von Wohnraum zur Wehr zu setzen. Dazu kamen, wie Kaltenborn in quellennaher Anlehnung an zeitgenössische amtliche Beurteilungen zeigt, auch selbstverschuldete Probleme der Vertriebenen, die keineswegs immer alle Möglichkeiten nutzten und durch teilweise überzogene Ansprüche das Verhältnis zu den Einheimischen zusätzlich beeinträchtigten. Insgesamt sei es der thüringischen Landespolitik lediglich gelungen, bis 1948 die allergrößte Not zu lindern. Die Vergabe wirklich menschenwürdigen Wohnraumes an Vertriebene einschließlich adäquater Ausstattung mit Mobiliar und Hausrat habe - im Unterschied zur offiziellen Propaganda - in der DDR selbst im Laufe der fünfziger Jahren noch nicht befriedigend verwirklicht werden können.

Im fünften Teil des Bandes geht es schließlich um Vertreibung und Integration als Lebens-Erfahrungen der Vertriebenen. Da für quantifizierende soziologische Integrationsstudien in der SBZ/DDR eine entsprechende Quellenbasis nicht vorhanden ist, sieht sich die Soziologie in ihren Bemühungen, die langfristigen Integrationsprozesse Vertriebener in der SBZ/DDR zu rekonstruieren, auf qualitative Mikrostudien verwiesen. In diesem Sinne versucht Ute Schmidt, Berlin, eine erfahrungsgeschichtliche Annäherung an die konkreten Bedingungen ländlicher Vertriebenenintegration am Beispiel der dörflichen Ansiedlung von Bessarabien-Deutschen in Mecklenburg. Die Integrationsbedingungen für diese Gruppe waren - im Vergleich zur großen Masse der Vertriebenen von verschiedenen Besonderheiten geprägt: Als besonders bedeutsam erweist sich die Tatsache, daß sie schon vor 1945, also in der NS-Zeit, „Umsiedler“-Status besaßen. Die Geschichte ihrer Entwurzelung reicht also wesentlich länger zurück, und Rückkehrhoffnungen waren noch wesentlich geringer als bei anderen Vertriebenen; nicht minder wichtig war die Tatsache, daß die Bessarabien-Deutschen nach 1945 nicht nur geschlossen in Dörfern siedelten, sondern innerhalb der neuen Lokalgesellschaften auch die Bevölkerungsmehrheit stellten, so daß sie auf lokaler und lebensweltlicher Ebene keine marginalisierte Minderheit darstellten, sondern in einem „Mehrheits-Ghetto" lebten. Vor diesem Hintergrund wird deutlich, daß die Integration auch dieser Vertriebenengruppe ein besonderer Langzeitprozeß war, der von den Beteiligten immer wieder neue Anpassungs- und Reflexionsanstrengungen erforderte, und die besonderen politischen Verhältnisse in der SBZ/DDR machten diese Eingliederung in Mecklenburg erst recht zu einem „steinigen Weg“. Dennoch blieben viele Bessarabiendeutsche in der SBZ/DDR und konnten sogar Landsleute, die bereits im Westen Fuß gefaßt hatten, zum Nachzug bewegen. Der Beitrag veranschaulicht nachdrücklich, daß in den Bessarabiendeutschen-Dörfern in Mecklenburg die traditionell eingeübten Verhaltensweisen und Formen dörflicher Selbstorganisation reaktiviert und weitgehend unterhalb der Konfliktschwelle praktiziert werden konnten. In politischer Hinsicht konnte die SED zwar die Mitglieder dieser Vertriebenengruppe nicht als loyale Klientel für sich gewinnen; parteilos oder durch die Mitgliedschaft in der (SED-konformen) DBD neutralisiert, waren die Bessarabiendeutschen in ihrer Mehrheit jedoch auch nicht oppositionell eingestellt, sondern zeigten sich weithin bereit, sich den gegebenen Verhältnissen anzupassen.

Einen weiteren, ganz anders gearteten Sonderfall der Vertriebenenintegration behandelt Dagmar Semmelmann, Berlin, aus erfahrungsgeschichtlicher Perspektive. Sie beschäftigt sich mit Vertriebenen, die - nach einer mehr oder weniger harten Übergangsbzw. Einstiegsphase bis etwa 1951/52 - ihre dauerhafte Integration und Sozialisation im sozialistisch-schwerindustriellen Großprojekt des „EKO“ (Eisenhüttenkombinat Ost) 
und der darum entstehenden „Stalinstadt" bzw. „Eisenhüttenstadt" erfuhren; die meisten von ihnen traten der SED bei, da sie dies als Teil ihrer Integration in Arbeits- und Lebenswelt begriffen. Semmelmann unternimmt den Versuch, den reibungs- und widerspruchsvollen Verlauf dieses Prozesses im biographischen Längsschnitt unter sich grundlegend verändernden gesellschaftspolitischen Bedingungen aus der Retrospektive der Befragten nachzuzeichnen und zu analysieren. Am Beispiel von sechs ausgewählten, zum Teil deutlich voneinander abweichenden Fallbeispielen wird der Zusammenhang von Vertreibungs- und Integrationserfahrung beleuchtet und der Prozeß der Eingliederung der Heimatvertriebenen in seinen verschiedenen Phasen nachvollzogen. Auf eine erste relativ kurze, der eigentlichen Integration "vorgelagerte“ und von Mangel und Not gekennzeichnete Periode (1945-1948) folgte bei den Befragten eine Umschwungoder Aufbruchperiode. Dabei gewann die Einbindung in den Arbeitsprozeß zentrale Bedeutung und förderte somit die soziale und politische Integration der Vertriebenen wesentlich. Anhand verschiedener Spielarten von SED-Beitritten veranschaulicht Semmelmann den Zusammenhang von Vertreibungserfahrung, individuell befriedigend verlaufender Eingliederung in den Arbeitsmarkt und wachsender Bereitschaft zur sozialen und politischen Einbindung in die neuen Verhältnisse, wie er sich bei diesen in einer Wachstumsregion der DDR lebenden und arbeitenden Vertriebenen entwickelte. Diese sahen - im Gegensatz zu jener beträchtlichen Minderheit, die bis 1961 nach Westen abwanderte - wie die Mehrheit der Vertriebenen in der DDR irgendwann keinen Grund mehr, „zu gehen“.

Der abschließende sechste Teil des Bandes präsentiert zwei laufende, regional fokussierte Forschungsprojekte zur Vertriebenenintegration nach 1945. Winfried Müller, München, stellt das vom Bayerischen Staatsministerium für Arbeit und Sozialordnung, Familie, Frauen und Gesundheit initiierte, an verschiedenen Universitäten des Landes Bayern angesiedelte Forschungsprojekt „Die Entwicklung Bayerns durch die Integration der Heimatvertriebenen und Flüchtlinge" vor. Unter der Forschungsprämisse "Wandel durch Integration" soll dabei den Fragen nachgegangen werden, welche Auswirkungen der Zustrom von mehr als zwei Millionen Vertriebenen und Flüchtlingen auf Bayerns Wirtschaft und Gesellschaft, auf Politik und Verwaltung, auf Kultur- und Geistesleben hatte, und welche Impulse für die Modernisierung des Aufnahmelandes Bayern von den Heimatvertriebenen und Flüchtlingen nach dem Neubeginn des Jahres 1945 ausgingen. Nach einer Vorstellung bereits vorliegender Publikationen skizziert Müller zwei 1995 begonnene Teilprojekte: a) Vereinswesen als Integrationsfaktor und b) Integration der Heimatvertriebenen und Flüchtlinge im Bildungssektor.

Das Sächsische Staatsministerium des Innern fördert an der Universität Leipzig unter Leitung von Ulrich von Hehl ein vierteiliges Projekt zur „Aufnahme und Integration von Flüchtlingen und Vertriebenen in Sachsen von 1945 bis 1952“, das in einem gemeinsam verfaßten Beitrag der vier Doktoranden des Projektes vorgestellt wird. Im ersten Teilprojekt werden die Grundsatzentscheidungen der Flüchtlings- und Vertriebenenpolitik der sowjetischen Besatzungsmacht und der SED und die damit verbundenen Auswirkungen auf die Tätigkeit der Landesregierung Sachsen untersucht. Damit eng verbunden ist das zweite Teilprojekt, das sich mit der administrativen Umsetzung der zentralen Vorgaben in ausgewählten sächsischen Städten und Kreisen beschäftigt. Das dritte Teilprojekt untersucht die Vertriebenenpolitik der LDP. Im Mittelpunkt stehen hier alternative und mit der Politik der SED konkurrierende Konzepte. Zur Ergänzung der vorwiegend 
politikgeschichtlich angelegten Teile stehen im vierten Teilprojekt Fragen nach dem Selbstbild und den Erfahrungsstrukturen der Vertriebenen im Vordergrund.

$* * *$

Die Literaturverweise in den Fußnoten sind einheitlich in Kurzform (Autor, Kurztitel, Seitenzahl) gehalten und können vollständig aus dem Gesamt-Literaturverzeichnis am Ende des Bandes erschlossen werden. Einschübe bzw. Auslassungen in Zitaten sind durch eckige Klammern kenntlich gemacht worden.

Für die Mitwirkung an der Gestaltung des Bandes danken die Herausgeber herzlich Frau Steffi Manske, Herrn Leonhard Hruschka sowie Herrn Olaf Blumenstein. Ein besonderer Dank gilt ferner dem bisherigen Leiter der Berliner Außenstelle des Instituts für Zeitgeschichte, Dr. habil. Hartmut Mehringer, sowie Dr. Michael Buddrus, die manche technischen Hindernisse bei der Erstellung dieses Bandes aus dem Weg räumen halfen.

$\mathrm{Zu}$ danken ist schließlich dem Bundesministerium des Innern, welches das Forschungskolloquium „Integration von Flüchtlingen im Nachkriegsdeutschland“ des Instituts für Zeitgeschichte im Juni 1996, auf das dieser Band zurückgeht, durch großzügige Förderung ermöglichte, sowie dem Bundesverwaltungsamt für die organisatorische $\mathrm{Ab}$ wicklung. 